\title{
Trends of Metastasis-Directed Treatments in Patients with Renal Cell Carcinoma: A Total Population-Based Analysis in Germany in the Era of Targeted Therapies
}

\author{
Christian P. Meyer ${ }^{\mathrm{a}}$ Christer Groeben ${ }^{\mathrm{b}}$ Phillip Marks ${ }^{\mathrm{a}} \quad$ Rainer Koch $^{\mathrm{b}}$ \\ Johannes Huber ${ }^{\text {b }}$ \\ aDepartment of Urology, University Medical Center Hamburg-Eppendorf, Hamburg, Germany; ${ }^{b}$ Department of \\ Urology, Medical Faculty Carl Gustav Carus, Technical University Dresden, Dresden, Germany
}

\section{Keywords}

Renal cell carcinoma - Metastasis - Metastasectomy ·

Radiotherapy $\cdot$ Health services research

\begin{abstract}
Introduction: We characterize trends of metastasis-directed treatments in patients with metastatic renal cell carcinoma (mRCC) in Germany in the targeted therapy era. Methods: We identified all cases with a diagnosis of renal cell carcinoma (ICD-10: C.64) and site-specific codes for secondary malignant neoplasms (C79.x) in combination with procedural codes for resection and radiation from the Institute of Hospital Remuneration and the German Federal Statistical Office (Destatis) between 2006 and 2014. We assessed site-specific temporal trends using estimated annual percent change (EAPC) linear regression. Results: Overall, 15,742 resections and 21,224 radiation treatments were recorded. These targeted lung ( $44.1 \%$ resections; $22.0 \%$ radiations), lymph node (14.0\% resections; $12 \%$ radiations), bone (21\% resections; $38 \%$ radiations), liver ( $9 \%$ resections; $7 \%$ radiations), adrenal ( $11 \%$ resections; $3 \%$ radiations), and CNS metastases ( $2 \%$ resections; $19 \%$ radiations). There was a significantly increasing trend for resection of lung (EAPC $+1.33, p=0.011)$, bone $(\mathrm{EAPC}+2.48, p=0.014)$, and adrenal $(\mathrm{EAPC}+3.4, p=0.003)$ metastases, while trends for resection of CNS metastases significantly decreased (EAPC -7.93, $p=0.005$ ). Between Western and Eastern Germany linear trends of resection (EAPC
\end{abstract}

$+2.75, p<0.001$; EAPC $-0.44, p=0.54$ ) and radiation (EAPC $+1.08, p=0.15 ;-3.41, p=0.03$ ) differed significantly. Conclusion: We observed an increasing trend for metastasis-directed resections and slightly declining numbers for radiation therapy in Germany for mRCC in the targeted therapy era. Treatment differed by geography. These findings suggest more aggressive treatment algorithms following the availability of targeted therapies and a yet diverging treatment landscape needing further exploration.

() 2020 S. Karger AG, Basel

\section{Introduction}

In 2020, an estimated 15,400 new cases of renal malignancies will be diagnosed in Germany, with approximately 5,400 new deaths [1]. While approximately $15 \%$ show synchronous metastases, almost $40 \%$ of patients will face a metastatic situation over time [2]. Metastatic spread most frequently occurs in the lungs (60-75\%), lymph nodes and bones (40\%), liver (19-40\%), adrenals (2$10 \%$ ), and the central nervous system (CNS; 5-7\%) [3]. Mortality has seen a dramatic decrease since the introduction of targeted therapies with a doubling of median survival across all prognostic groups [4-7]. Despite pharmacological advances, local treatment of metastases with

C.P.M. and C.G. contributed equally to this work. 
radiation or surgery remains the only therapeutic option with curative intent. Moreover, it sometimes offers an effective palliative treatment. Although retrospective series have shown a significant survival benefit of $14-17 \%$ when metastasectomy was performed in the targeted therapy era $[8,9]$, the exact status of metastasis-directed therapy in the treatment algorithm of metastatic renal cell carcinoma (mRCC) has been poorly explored [10]. Recently, Lyon et al. [11] have reported favorable cancer specific outcomes after complete resection of metastases in RCC in the post-cytokine era, but reports on the dissemination of metastasis-directed therapies on a population-based level are scarce. However, it is important to complement evidence from clinical, retrospective studies with population-based data to allow for a more generalizable assessment [12].

In the current study, we sought to characterize the situation of metastasis-directed therapy in mRCC in Germany during the targeted therapy era from 2006 to 2014 .

\section{Materials and Methods}

Since 2004, reimbursement of inpatient treatment in Germany is based on an adapted version of the international diagnosis related groups (DRG) coding diagnoses according to ICD-10 and medical procedures according to a German version of the international classification of operations and procedures. Records containing the diagnoses and procedures of each in-hospital case are transferred annually to the Institute of Hospital Remuneration and consecutively to the German Federal Statistical Office (Destatis). Because of German data protection requirements, access to the raw data is prohibited and must be performed by remote data processing. Likewise, identification of single cases or hospitals is not possible and results, which could allow identification, are censored.

We performed all actions in accordance with the Declaration of Helsinki in its latest version and adhered to the RECORD (Reporting of studies Conducted using Observational Routinely collected health Data) statement [13]. Analyzed data were completely anonymized and derived from an established database with rigorous data protection measures.

We identified all cases with a primary diagnosis of renal cell carcinoma (ICD-10: C.64) and site-specific codes for secondary malignant neoplasms (C77-79.ff) in combination with procedural codes for resection and radiation. Since radiation codes do not explicitly discriminate for specific organ sites, only those radiation procedures simultaneously coded along with the main and sitespecific secondary diagnosis codes were considered for further analysis. For example, for lung radiation, we considered only those cases with a concomitant diagnosis of renal cell cancer (C64), a secondary neoplasm of the lung (C78) and the OPS procedural codes for radiotherapy (OPS Codes 8-52ff.) This search strategy sought to identify treatments on a case but not patient basis. However, as there are no site-specific radiotherapy codes, we additionally calculated cross-tables of sites to identify the proportions of potential multiple orang-site counting.

Descriptive statistics focused on frequencies and proportions. Proportions were stratified according to gender and geographic location (East vs. West). Finally, we assessed site-specific temporal trends using estimated annual percent change (EAPC) linear re- 
Fig. 1. Proportions of location in patients with mRCC between 2006 and 2014 stratified by treatment.
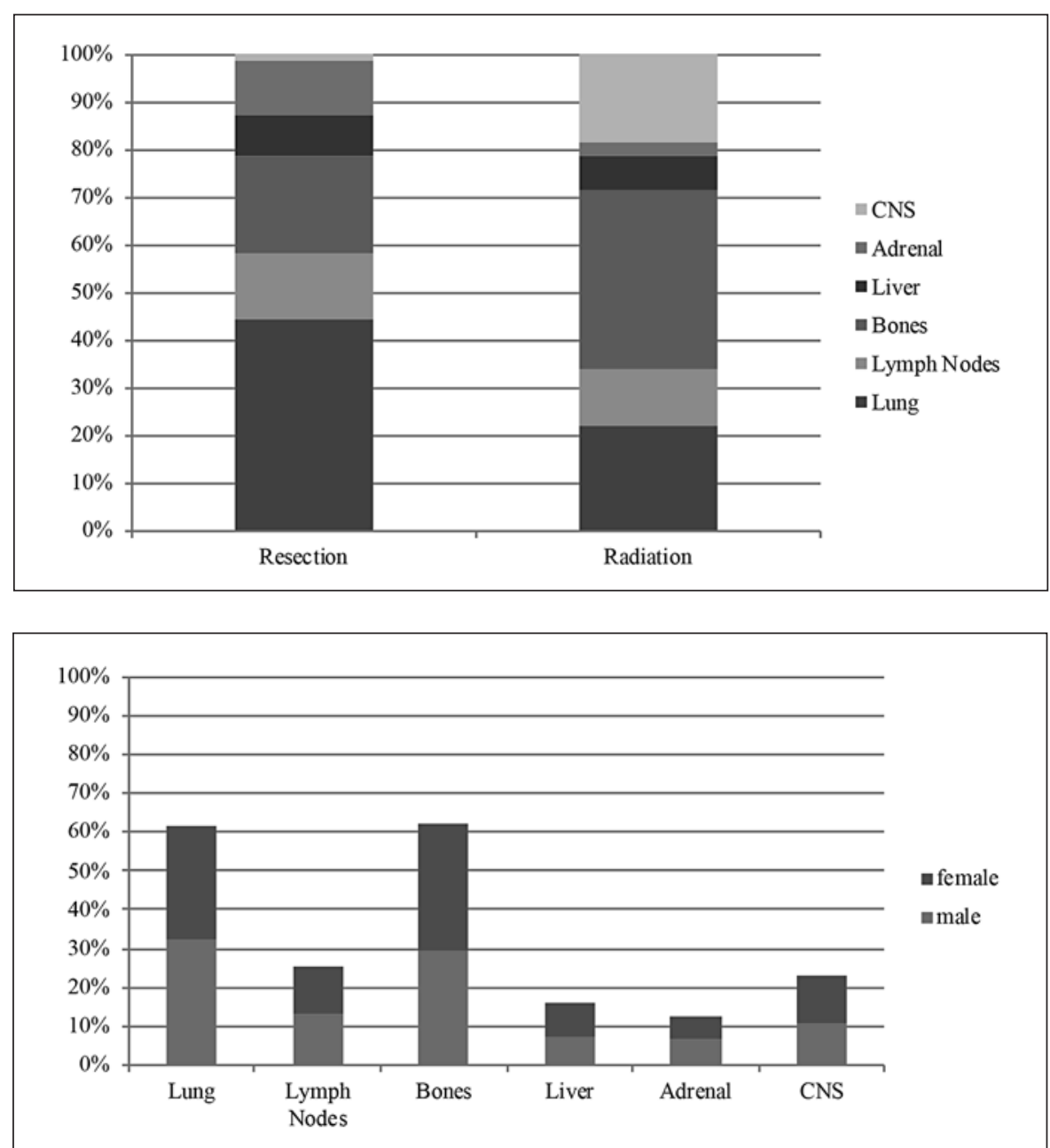

Fig. 2. Proportions of gender by site in mRCC patients with metastasis-directed therapy between 2006 and 2014. gression as previously described $[14,15]$. We defined $p<0.05$ to indicate significance. SAS 9.4 (SAS Institute $\mathrm{GmbH}$, Heidelberg, Germany) was used for all analyses.

\section{Results}

We identified 36,951 cases with a metastasis-directed therapy, of which $15,727(42.6 \%)$ were resections and $21,224(57.4 \%)$ radiation therapy. The majority of cases were male $(n=24,779,67.1 \%)$. Mean (median) age was 67.3 (65) years. The number of resections versus radiations were 6,931 versus 4,677 in lung, 2,195 versus 2,489 in lymph nodes, 3,246 versus 8,023 in bone, 1,370 versus 1,502 in liver, 1,749 versus 603 in adrenal, and 236 versus 3,930 in CNS metastasis (Table 1; Fig. 1, 2). Of radiated patients, $5.6 \%$ had a concomitant coding for bone and CNS metastasis, 9.9\% for bone and lung metastasis. There was a significantly increasing trend for resection of lung $(\mathrm{EAPC}+1.33, p=0.011)$, bone $(\mathrm{EAPC}+2.48, p=0.014)$, and adrenal $(\mathrm{EAPC}+3.4, p=0.003)$ metastasis, while trends for resection of CNS metastasis significantly de- creased $($ EAPC $-7.93, p=0.005)$ or did not change (lymph nodes, $\mathrm{EAPC}+1.19, p=0.12$ ) over time. Radiation was the mainstay of therapy for bone (EAPC $-0.1, p=0.86$ ) and CNS (EAPC $-0.61, p=0.53$ ) metastasis and was stable over time. There was no significant change for radiation to lung (EAPC $-0.16, p=0.84$ ), lymph node (EAPC $0.7, p=0.48$ ), liver (EAPC $-0.1, p=0.92$ ), and adrenal $(\mathrm{EAPC}+2.95, p=0.07)$ metastasis (Fig. 3). Comparing Western versus Eastern Germany, there were a total of 10,855 versus 4,872 resections and 15,479 versus 5,745 radiation therapies. Trends were geographically significantly different in Western versus Eastern Germany for resection $(\mathrm{EAPC}+2.75, p<0.001$; EAPC $-0.44, p=0.54)$ and radiation $(\mathrm{EAPC}+1.08, p=0.15 ;-3.41, p=0.03)$, respectively (Fig. 4).

\section{Discussion}

Against the backdrop of limited systemic options, metastasis-directed therapy has been a stable factor in the treatment of mRCC for decades. Most evidence is still 


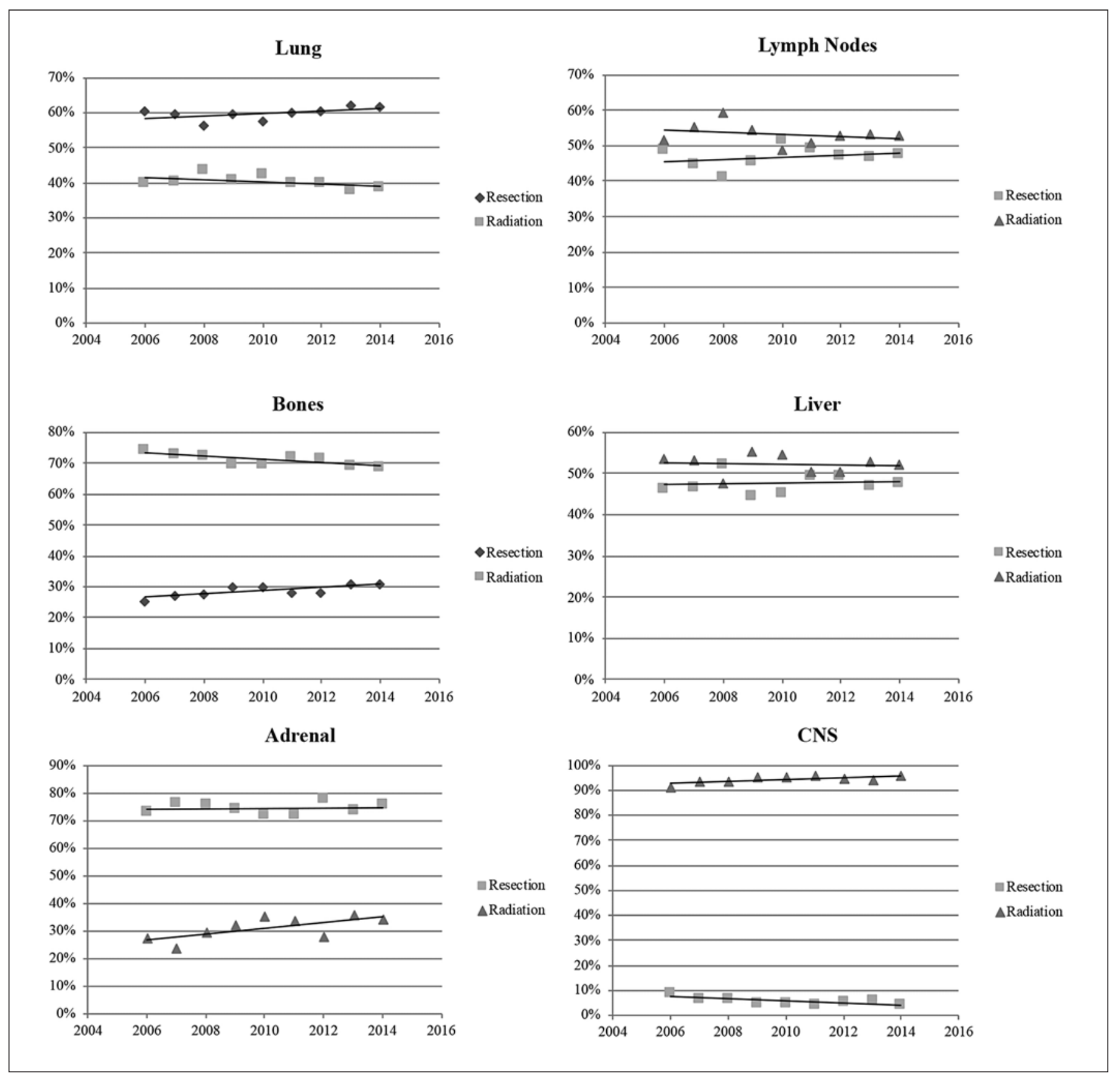

Fig. 3. Linear trends of metastasis-directed resection and radiation in mRCC between 2006 and 2014 by site.

from the cytokine era or from small contemporary series. Only recently, evidence from population-based studies in the targeted therapy era have been emerging on the applicability and safety of metastatic resections $[9,16]$. This, however, is important in order to understand and generalize findings on a larger scale. In this regard, our study holds several important findings.

First, we identified a significant surge in metastasisdirected therapy, but especially surgical resections over the study period. This is in line with data from North America, where an EAPC of $+2.18 \%$ /year was noticed be- tween 2006 and 2013 [9]. Moreover, there seems to be a more generous use of aggressive surgical treatment across other solid tumor entities. For example, Bartlett et al. [17] reported a steady increase of metastasectomy for gastrointestinal tumors between 2000 and 2011. In RCC, metastasis-directed therapy is considered the only treatment with curative intent when a complete resection can be achieved [8]. However, there is a strong heterogeneity between sites of metastasis. For example, complete resection of solitary lung metastases resulted in an excellent 5 -year cancer specific survival rate of $73.6 \%$ in the Mayo- 

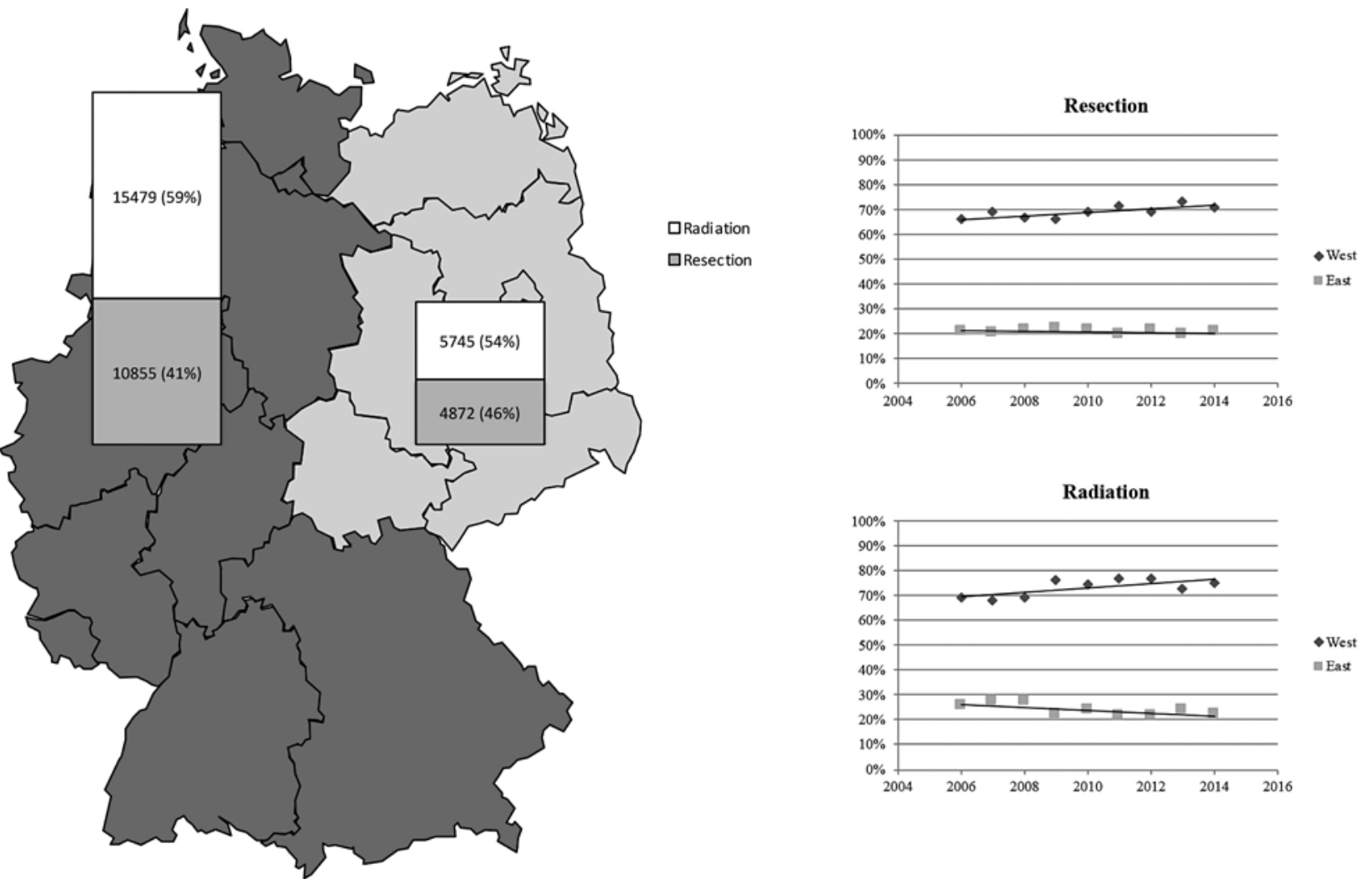

Fig. 4. Geographic differentiation and trends for resection and radiation of mRCC between 2006 and 2014.

Clinic cohort between 1979 and 2006 in solitary metastasis. This dropped to $19 \%$ when only an incomplete resection could be achieved [18]. Other prognostic factors for long-term survival were negative lymph nodes, a metachronous metastasis, and the number of metastases. Although systemic therapies, either in neo-adjuvant or adjuvant intention, have so far failed to confer a significant benefit in conjunction with surgical resection, the significant increase in lung resections over the study period might be the consequence of increased responses to targeted therapies.

In contrast, outcomes for aggressive surgical removal of bone metastases are poor. Besides oncological indications, these surgeries can be necessary due to functional or symptomatic problems. In a comprehensive review, Mir et al. [3] report a comparatively meager 5-year survival of only $10-35 \%$ in solitary metastases, and of $0 \%$ with multiple bone metastases. In combination with targeted therapies, the surgical resection can increase the oncological outcome from 7.6 to 31.8 months, although the functional or symptomatic effect could not be assessed [19]. The favorable combination of systemic therapy and surgical removal might have triggered the significant increase of bone resections in our study cohort.
As reflected by our results, radiation is the treatment of choice for CNS metastases. Although, both, a complete resection or stereotactic radiation, have proven successful in local control and cure, CNS metastasis are associated with a generally poor overall outcome, for example, a 5 -year survival of $12 \%$ [20]. Due to their multifocality, they are most often not easily amendable to surgery [3, 21].

Especially for lung metastases (12\%), we saw a higher proportion of radiation therapy than expected. While comparative epidemiological data is not available, clinical studies do confirm a non-negligible number of radiations (conventional and stereotactic) [22, 23]. Nevertheless, from a clinical perspective, these treatments seem to be last resorts for otherwise not manageable disease.

Against the backdrop of favorable outcomes with metastasis-directed therapy, it needs to be stressed that surgical resections of metastases in RCC have non-negligible complication rates of up to $27 \%$. This matches complication rates of radiation with up to $25 \%$ and systemic adverse events of targeted therapies and immune oncology $[16,24,25]$.

Second, we observed a significant difference in regional trends of metastasis-directed therapy in Germany. 
While the distribution of treatments corresponds to the per capita population of Eastern and Western Germany, there are surprisingly diverging trends with significantly increasing resections in Western Germany and decreasing trends in Eastern Germany. Although final conclusions cannot be drawn on the basis of our data, our results may be indicative of lingering regional differences that have been reported for years. Despite a generally broad access to care through statutory health plans and private health insurance, several issues have emerged. For years, regional redistributions have altered population agebrackets and continue to do so. For example, the percentage of elderly people $>60$ years and $>80$ years will have surged by 15 and $26 \%$ by 2025 . This shift will mostly impact the north-eastern parts of Germany. Consequently, these regions are expected to lead cancer incidence rates by 2025, a prognosis published by the Germany Association of Hematology and Oncology [26]. Explanations of regional health disparities have further been based on the interaction of supply-sensitive, preference-sensitive, and effective care [27]. Effective care to that extent might also include the rigorous implementation of centers of expertise and cross-regional multidisciplinary tumor boards. In short, these concepts describe deviations from perfect regional health care systems, where demand and patient preferences are synchronously matched. To that extent, our data provide evidence that these problems do not only concern widespread diseases but do have significant effect on less common cancers like mRCC as well.

Our study is not devoid of limitations. First, as with other retrospective data, we could not account for unmeasured confounders, especially the accuracy of coding within the database. However, as the data is used for reimbursement, coding adherence is intrinsically prudent. Second, it is important to realize that numbers are cases and do not represent individual patients, that is, a patient might have received more than one metastasis-directed therapy. Although no multiple counting by organ-site in the radiation cohort accounted for more than $10 \%$ of cases, numbers might have been inflated by up to that percentage due to unspecific radiation therapy classifiers in the database. Furthermore, the design of the database only allows for analysis of inpatients. Therefore, especially patients receiving radiotherapy in an outpatient setting could not be detected. Since there is little granular and clinical information within the database, a more detailed assessment was not possible. Consequently, we could not establish whether a treatment in our cohort was for curation or palliation. Moreover, there is no information on the case histories, and we cannot analyze subgroups like synchronous or metachronous metastatic spread. Third, the study time frame almost exclusively captures the targeted therapy era, and current standard practice is overhauled by immune-oncology. Thus, it may not represent the current treatment landscape. At this time, it is unclear whether the improved responses to modern immune therapy will lead to even more aggressive surgical approaches or will attenuate this effect.

\section{Conclusion}

Our data show an increase in surgical therapies, most probably due to more aggressive treatment algorithms in accordance with current guideline recommendations. Targeted therapies might have expanded the indications for such approaches on the basis of encouraging combined treatment algorithms and extended survival periods. It is evident that potential candidates for such treatments need an experienced and interdisciplinary team to ensure adequate patient selection and optimal treatment sequences [28]. In the wake of the emerging immune oncological options for mRCC patients, the role of metastasis-directed therapy needs to be continuously evaluated.

\section{Acknowledgments}

Research data centers of the federal and state statistical offices, DRG-statistics 2006 to 2014, own calculations.

\section{Statement of Ethics}

We performed all actions in accordance with the Declaration of Helsinki in its latest version and adhered to the RECORD (Reporting of studies Conducted using Observational Routinely collected health Data) statement. Analyzed data were completely anonymized and derived from an established database with rigorous data protection measures.

\section{Conflict of Interest Statement}

The authors have no conflicts of interest to declare.

\section{Funding Sources}

Med-Drive Grant of the Medical Faculty Carl Gustav Carus, TU Dresden (Grant No.: 60.356).

\section{Author Contributions}

C.P.M.: protocol/project development, data analysis, manuscript writing. C.G. and J.H.: data collection and management, data analysis, manuscript editing. P.M.: project development, manuscript editing. R.K.: data collection and management, manuscript editing. 


\section{References}

1 Fiebig J, Kraywinkel K. Epidemiologie des Nierenzellkarzinoms in Deutschland. Der Onkologe. 2019;25(6):483-87.

2 Rini BI, Campbell SC, Escudier B. Renal cell carcinoma. Lancet. 2009 Mar;373(9669): 1119-32.

3 Mir MC, Matin SF, Bex A, Spiess PE, Thompson RH, Grob B, et al. The role of surgery in the management of metastatic kidney cancer: an evidence-based collaborative review. $\mathrm{Mi}$ nerva Urol Nefrol. 2018 Apr;70(2):109-25.

4 Motzer RJ, Hutson TE, Tomczak P, Michaelson MD, Bukowski RM, Rixe O, et al. Sunitinib versus interferon alfa in metastatic renal-cell carcinoma. N Engl J Med. 2007 Jan; 356(2):115-24.

5 Motzer RJ, Hutson TE, Tomczak P, Michaelson MD, Bukowski RM, Oudard S, et al. Overall survival and updated results for sunitinib compared with interferon alfa in patients with metastatic renal cell carcinoma. J Clin Oncol. 2009 Aug;27(22):3584-90.

6 Heng DY, Xie W, Regan MM, Harshman LC, Bjarnason GA, Vaishampayan UN, et al. External validation and comparison with other models of the International Metastatic RenalCell Carcinoma Database Consortium prognostic model: a population-based study. Lancet Oncol. 2013 Feb;14(2):141-8.

7 Albiges L, Choueiri T, Escudier B, Galsky M, George D, Hofmann F, et al. A systematic review of sequencing and combinations of systemic therapy in metastatic renal cancer. Eur Urol. 2015 Jan;67(1):100-10.

8 Dabestani S, Marconi L, Hofmann F, Stewart F, Lam TB, Canfield SE, et al. Local treatments for metastases of renal cell carcinoma: a systematic review. Lancet Oncol. 2014 Nov; 15(12):e549-61.

9 Sun M, Meyer CP, Karam JA, de Velasco G, Chang SL, Pal SK, et al. Predictors, utilization patterns, and overall survival of patients undergoing metastasectomy for metastatic renal cell carcinoma in the era of targeted therapy. Eur J Surg Oncol. 2018 Sep;44(9):1439-45.

10 Kim DY, Karam JA, Wood CG. Role of metastasectomy for metastatic renal cell carcinoma in the era of targeted therapy. World J Urol. 2014 Jun;32(3):631-42.
11 Lyon TD, Thompson RH, Shah PH, Lohse CM, Boorjian SA, Costello BA, et al. Complete Surgical Metastasectomy of Renal Cell Carcinoma in the Post-Cytokine Era. J Urol. 2020 Feb;203(2):275-82.

12 Flegar L, Groeben C, Koch R, Baunacke M, Borkowetz A, Kraywinkel K, et al. Trends in Renal Tumor Surgery in the United States and Germany Between 2006 and 2014: Organ Preservation Rate Is Improving. Ann Surg Oncol. 2020 Jun;27(6):1920-28.

13 Benchimol EI, Smeeth L, Guttmann A, Harron K, Moher D, Petersen I, et al.; RECORD Working Committee. The REporting of studies Conducted using Observational Routinely-collected health Data (RECORD) statement. PLoS Med. 2015 Oct;12(10):e1001885.

14 Estève J, Benhamou E, Raymond L. Statistical methods in cancer research. Volume IV. Descriptive epidemiology. IARC Sci Publ. 1994;(128):1-302.

15 Sammon JD, Pucheril D, Abdollah F, Varda B, Sood A, Bhojani N, et al. Preventable mortality after common urological surgery: failing to rescue? BJU Int. 2015 Apr;115(4):666-74.

16 Meyer CP, Sun M, Karam JA, Leow JJ, de Velasco G, Pal SK, et al. Complications After Metastasectomy for Renal Cell Carcinoma-A Population-based Assessment. Eur Urol. 2017 Aug;72(2):171-4.

17 Bartlett EK, Simmons KD, Wachtel H, Roses RE, Fraker DL, Kelz RR, et al. The rise in metastasectomy across cancer types over the past decade. Cancer. 2015 Mar;121(5):747-57.

18 Alt AL, Boorjian SA, Lohse CM, Costello BA, Leibovich BC, Blute ML. Survival after complete surgical resection of multiple metastases from renal cell carcinoma. Cancer. $2011 \mathrm{Jul}$; 117(13):2873-82.

19 Ouzaid I, Capitanio U, Staehler M, Wood CG, Leibovich BC, Ljungberg B, et al.; Young Academic Urologists Kidney Cancer Working Group of the European Association of Urology. Surgical Metastasectomy in Renal Cell Carcinoma: A Systematic Review. Eur Urol Oncol. 2019 Mar;2(2):141-9.
20 Shuch B, La Rochelle JC, Klatte T, Riggs SB, Liu W, Kabbinavar FF, et al. Brain metastasis from renal cell carcinoma: presentation, recurrence, and survival. Cancer. 2008 Oct; 113(7):1641-8.

21 Sperduto CM, Watanabe Y, Mullan J, Hood T, Dyste G, Watts C, et al. A validation study of a new prognostic index for patients with brain metastases: the Graded Prognostic Assessment. J Neurosurg. 2008 Dec;109(Suppl): 87-9.

22 Stinauer MA, Kavanagh BD, Schefter TE, Gonzalez R, Flaig T, Lewis K, et al. Stereotactic body radiation therapy for melanoma and renal cell carcinoma: impact of single fraction equivalent dose on local control. Radiat Oncol. 2011 Apr;6(1):34.

23 Ranck MC, Golden DW, Corbin KS, Hasselle MD, Liauw SL, Stadler WM, et al. Stereotactic body radiotherapy for the treatment of oligometastatic renal cell carcinoma. Am J Clin Oncol. 2013 Dec;36(6):589-95.

24 Leitlinienprogramm Onkologie (Deutsche Krebsgesellschaft DK, AWMF): Diagnostik, Therapie und Nachsorge des Nierenzellkarzinoms, Langversion 2.01,2020, AWMF Registernummer: 043/017OL.

25 Wersäll PJ, Blomgren H, Lax I, Kälkner KM, Linder C, Lundell G, et al. Extracranial stereotactic radiotherapy for primary and metastatic renal cell carcinoma. Radiother Oncol. 2005 Oct;77(1):88-95.

26 Deutschlandweite Prognose der bevölkerungsbezogenen Morbiditätserwartung für häufige Krebserkrankungen - Auswirkungen auf die Versorgung. Gesundheitspolitische Schriftenreihe der DGHO: Deutsche Gesellschaft für Hämatologie und Onkologie.

27 Wennberg J. Tracking Medicine. Oxford: Oxford University Press; 2010.

28 Huber J, Ihrig A, Winkler E, Brechtel A, Friederich HC, Herzog W, et al. Interdisciplinary counseling service for renal malignancies: a patient-centered approach to raise guideline adherence. Urol Oncol. 2015 Jan;33(1):23.e1e7. 